\title{
Enseigner la relation médecin-malade Présentation d'une expérience pédagogique développée à la faculté de médecine de Créteil
}

\author{
Teaching doctor-patient relationship :presentation of a pedagocigal experience developed at the \\ Faculty of medicine of Créteil.
}

Guy EVEN*

Résumé Contexte : La formation à la relation médecin-malade requiert l'utilisation de dispositifs pédagogiques et de méthodes d'apprentissage spécifiques. Elle fait l'objet d'un développement progressif depuis une vingtaine d'années à la faculté de médecine de Créteil- Paris XII. Cadre conceptuel et description : Le programme d' "enseignement d'initiation à la relation médecin malade "s’appuie sur un ensemble de principes pédagogiques éclectiques qui visent à prendre en compte à la fois des éléments cognitifs et des éléments émotionnels. Il recourt à des moyens et à des ressources pédagogiques variés : interventions dès les premiers contacts avec les patients, travail de formation s'appuyant sur l'expérience des étudiants dans les services hospitaliers, utilisation du groupe de parole sur cas et du jeu de rôle co-animés par un médecin et un psychologue, psychiatre, psychanalyste, écriture d’un texte avec références bibliographiques. Résultats et évaluation : Les étudiants ont fait part de façon récurrente d'un taux élevé de satisfaction ; leur participation et leurs travaux personnels sont jugés positivement par les enseignants. L'ensemble du dispositif a montré sa viabilité opérationnelle.

Mots clés Relation médecin malade ; dispositif pédagogique de formation ; groupes de parole ; cas cliniques ; jeu de rôle.

Abstract Context: Doctor-patient relationship training requires the use of specific pedagogical systems and learning methods. During the past 20 years, this formation has been developed progressively at the Faculty of medicine of Créteil-Paris XII. Conceptual framework and methods: Teaching program introducing doctor-patient relationship formation relies on eclectic pedagogical principles based on cognitive as well as emotional elements. This program uses various pedagogical means and resources such as: immediate interventions during first contacts with patients, work formation based from students experiences during their hospital duties, the formation of focus groups discussing on clinical cases, role play co-animated by a physician along with either a psychologist, a psychiatrist or a psychoanalyst and finally text writing with inclusion of bibliographical references. Results and evaluation: Students have reported a recurrent high level of satisfaction; teachers have positively judged their implication and personal work. The whole pedagogical system has demonstrated its practicability.

Key words Doctor-patient relationship; training pedagogical system; clinical cases; role play.

Pédagogie Médicale 2006;7:165-73

${ }^{*}$ Coordonnateur - Département d'enseignement et de recherche en psychologie médicale - Faculté de médecine de Créteil (Université de Paris XII, France). Animateur de groupe Balint (Association de recherche et d'étude pour la formation à la fonction soignante -AREFFSet Société médicale Balint -SMB-)

Correspondance : Guy Even - 20 rue Jean Jaurès - 94500 Champigny sur Marne - France - Téléphone : + 33 (1) 49830830

Mailto:getg.even@wanadoo.fr 


\section{Introduction}

Jusqu'à une date assez récente, la formation à la relation médecin-malade - traditionnellement considérée comme relevant de l'expérience de chaque médecin - ne faisait l'objet, dans la plupart de nos facultés de médecine, d'aucun programme d'enseignement structuré. La relative nouveauté de ce secteur d'enseignement, la diversité des dispositifs pédagogiques proposés, les différentes réponses apportées par chaque équipe d'enseignants aux questions concernant les contenus transmissibles à privilégier, les méthodes pédagogiques à utiliser, les évaluations réalisables, invitent à se situer dans une dynamique de recherche où l'échange d'expériences concrètes et l'explicitation détaillée des méthodes utilisées par chacun occupent une place de choix. Cet article est consacré à la description d'un tel dispositif de formation, mis en place depuis l'année 2001 au sein de la faculté de médecine de Créteil (Université de Paris XII), sous forme d'un " enseignement d'initiation à la relation médecin malade ».

\section{Contexte institutionnel}

Au début des années 70 , un enseignement de psychologie médicale, dont était chargé l'enseignant hospitalo-universitaire chef de service de psychiatrie, a été institué à la faculté de médecine de Créteil en seconde année du premier cycle des études médicales (PCEM2). Très rapidement celui-ci décida d'adjoindre à son cours magistral des séances d'enseignement dirigé, dont il confia l'organisation à un groupe de médecins généralistes intéressés. Il apparut rapidement que cette formation préliminaire n'était pas suffisante et que, dès la première année du deuxième cycle des études médicales (DCEM1) - c'est-àdire la troisième année du cursus -, nombre d'étudiants étaient en difficulté lors de leurs premières immersions dans le milieu hospitalier. Parallèlement aux enseignements dirigés, des groupes de parole et de réflexion furent alors mis en place, réunissant chacun une quinzaine d'étudiants autour de deux médecins généralistes puis, dès l'année suivante, d'un généraliste et d'un " professionnel de la psychologie clinique » cette dénomination, utilisée au sens générique, désignant aussi bien des psychiatres que des psychologues, analystes ou non, pourvu qu'ils aient une activité clinique. Le succès de ces groupes de travail fut tel qu'ils furent pérennisés sous la forme d'un programme d'enseignement facultatif, puis obligatoire, pour tous les étudiants de DCEM1, intitulé " enseignement d'initiation à la relation médecin-malade ». Ce programme est placé sous la responsabilité du Département d'enseignement et de recherche en psychologie médicale
(DERPM), qui rassemble actuellement plus de 30 enseignants, pour moitié médecins et pour moitié professionnels de psychologie clinique de diverses obédiences.

\section{Cadre conceptuel du programme d'initiation à la relation médecin-malade}

Dans l'élaboration et la mise en place d'un programme d'enseignement, des choix sont effectués par les équipes pédagogiques, certains positionnements théoriques étant privilégiés au détriment d'autres. Ces choix sont déterminés par le contexte et l'écologie de chaque faculté, ainsi que par les travaux déjà réalisés au sein de chaque équipe, qui font référence, de façon plus ou moins explicite, à des paradigmes déterminés. Les approches les plus récentes qui inspirent les travaux consacrés à l'enseignement et à l'apprentissage de la relation médecin-malade insistent sur la nécessité de ne pas confondre l'enseignement de la communication professionnelle et celui de la psychologie de la santé $^{1}$. Reconnaitre ce point de vue n'implique pas, selon nous, de négliger les apports de la psychologie clinique à la compréhension de la relation médecin-malade, et notamment de ceux issus de la perspective psychanalytique et du courant développé à partir des travaux de Balint. Nous formulons ci-après notre cadre conceptuel sous forme de sept thèses, en proposant au lecteur de les considérer pour ce qu'elles sont, une tentative visant à rendre compte des choix pédagogiques assumés et invitant à l'ouverture à un débat. A chacune de ces thèses est associé un principe pour l'enseignement de la relation médecin malade.

- Première thèse : pour un médecin, le fait de se situer « dans la lutte rationnelle contre la maladie » ou " dans la prise en charge relationnelle du sujet malade ", ne fait pas appel aux mêmes types "d'attentions et attitudes ${ }^{2}$ ", ni aux mêmes démarches psychiques et cognitives.

Premier principe pour l'enseignement : la formation à ces deux domaines hétérogènes de la pratique médicale requiert des méthodes pédagogiques radicalement différentes.

- Seconde thèse : dans le domaine relationnel, la formation des médecins passe essentiellement par l'expérience de la relation au malade et par la réflexion sur cette expérience.

Second principe pour l'enseignement : une formation à la relation médecin-malade doit inclure dans son dispositif pédagogique une expérimentation de la relation et la prise de recul réflexif sur celle-ci. 
- Troisième thèse : le début des études médicales est marqué par une forte identification des étudiants aux patients ${ }^{3}$ qui peut être source de traumatismes psychiques et/ou mise à profit pour leur formation à la relation.

Troisième principe pour l'enseignement : une intervention précoce dans les études médicales ouvre la possibilité de tirer profit du mouvement d'identification des étudiants au patient, de l'exploiter pédagogiquement et d'atténuer le traumatisme des premières rencontres à l'hôpital.

- Quatrième thèse : si, dans sa démarche clinique, le médecin utilise essentiellement des mécanismes de compréhension intellectuels, pour la prise en charge psychologique et relationnelle du patient, l'utilisation d'une « compréhension de nature émotionnelle $~^{4}$ venant s'adjoindre à la « compréhension intellectuelle» est nécessaire.

Quatrième principe pour l'enseignement : les éléments émotionnels mis en jeu dans la rencontre des étudiants avec les malades doivent être reconnus à leur juste valeur et pris en compte dans le dispositif de formation à la relation.

- Cinquième thèse : la capacité humaine de s'identifier à l'autre (en situation médicale, du médecin à «s'identifier au patient " ou à "se projeter en situation de malade ») étant à l'origine de cette " compréhension émotionnelle ", la façon dont s'installent pendant les études médicales les processus défensifs liés à cette identification et les manières de réagir aux confrontations difficiles auxquelles expose le métier peuvent avoir un effet considérable sur la pratique professionnelle relationnelle future du médecin.

Quatrième principe pour l'enseignement : une formation à la relation médecin malade doit favoriser la reconnaissance de l'existence des processus défensifs pour permettre que ceux-ci s'inscrivent de la manière la plus satisfaisante possible chez chaque étudiant.

- Sixième thèse : pour que l'identification au patient soit utilisable professionnellement, une formation favorisant la reconnaissance des éléments émotionnels et inconscients actifs du côté du médecin doit se doubler de l'acquisition de connaissances sur les processus psychiques en jeu dans la relation. Ces éléments de savoir, proposés aux étudiants comme un moyen de mieux comprendre leur expérience, fournissent des repères pour la relation.

Sixième principe pour l'enseignement : une formation à la relation médecin-malade doit donner aux étudiants des outils conceptuels leur permettant d'évaluer leur place dans la relation dès qu'ils en font l'expérience.

- Septième thèse : la place occupée par la subjectivité des protagonistes, la singularité de chaque expérience relation- nelle et la complexité des processus en ouvre dans la rencontre médecin-malade ont pour corollaire l'impossibilité de se référer pour la relation médecin-malade à des " modèles-types » de comportement à adopter par tous. Septième principe pour l'enseignement : dans la formation à la relation médecin-malade, «l'apprentissage des conduites à tenir » (sous entendu : pour tous) cède le pas à "l'interrogation sur les modes de réactions" (toujours d'abord singuliers et à partir desquels peuvent se repérer certaines régularités), dont il s'agit de repérer les limites et de soutenir les potentialités.

\section{Description du programme d'initiation à la relation médecin-malade}

L'enseignement proposé intervient en début des études médicales (DCEM1). Il concerne tous les étudiants d'une promotion et fait suite à six mois de stage dans les services hospitaliers. La présence y est obligatoire. Il est considéré comme un stage (les étudiants sont " en stage dans le groupe »). Les étudiants sont répartis en groupes de moins de vingt personnes. Ces groupes sont fixes pour l'ensemble de la séquence d'enseignement qui comporte 12 séances de deux heures chacune, au rythme d'une séance par semaine. Un premier module (" groupe de parole et de réflexion sur cas »), comportant six séances, est consacré au travail à partir des premières situations rencontrées par les étudiants à l'hôpital. Un second module (6 séances) utilise le " jeu de rôle " comme technique pédagogique. L'animation des groupes est assurée par un binôme d'enseignants, l'un étant médecin généraliste (ou spécialiste), l'autre professionnel de la psychologie clinique (psychiatre, psychologue, psychanalyste). Un " polycopié », constitué d'une compilation d'articles sur la relation médecinmalade est remis aux étudiants. Centré sur les concepts de base de la relation, il est conçu de manière à pouvoir être lu et intégré par l'ensemble des étudiants. A la fin des 12 séances d'enseignement, les étudiants rédigent un mémoire sur la relation médecin-malade à partir de leur expérience de formation dans les groupes.

\section{Objectifs du programme de formation}

Les objectifs généraux de cet enseignement sont les suivants : 1) prendre conscience de la dimension humaine et relationnelle de la pratique médicale ; 2) repérer la dynamique qui se développe entre les protagonistes de l'acte médical et susciter un travail de réflexion sur les interactions dans la relation médecin-malade ; 3 ) aider l'étudiant à percevoir et à entendre, au-delà du symptôme présenté par la personne malade, le sens de la démarche et 
de la demande du patient ; 4) préparer les étudiants à leur fonction de soignant. Il est précisé qu'il ne s'agit pas de proposer aux étudiants un modèle positif $\mathrm{du}$ " bon médecin " mais de leur ouvrir un lieu et un temps de réflexion leur permettant de se former à l'analyse de la dynamique de la relation médecin-malade.

Des objectifs spécifiques sont assignés au travail de groupe : 1) écouter les autres pour apprendre à écouter le patient ; 2) permettre une reconnaissance mutuelle des participants par la mise en place d'un lieu, d'un temps et d'une dynamique de groupe devant un problème identifié chez un patient, formuler de multiples hypothèses afin d'y adapter ses réponses ; 3 ) identifier les différentes facettes de son propre fonctionnement dans l'exercice médical (fonctionnement intellectuel et fonctionnement affectif) ; 4) identifier les distorsions dans l'écoute, l'examen et la relation.

D'autres objectifs spécifiques sont propres au jeu de rôle : 1) prendre conscience du ressenti du patient dans certaines situations ; 2) prendre conscience de l'effet des attitudes du soignant sur son interlocuteur ; 3) prendre conscience, en s'observant soi-même, de ses attitudes spontanées.

\section{Outils et ressources pédagogiques de base utilisés au cours du programme de formation}

Le dispositif pédagogique s'appuie sur quatre outils principaux : le cas, le groupe, le binôme enseignant, l'écriture d'un mémoire référencé.

\section{Le cas}

Chaque séance d'enseignement s'organise exclusivement autour de cas cliniques. Cette utilisation de situations cliniques concrètes, directement issues de la pratique, a comme intérêt : a) de démarquer l'enseignement de la "discussion à thème », avec sa propension à se perdre dans la généralité et le débat d'idée ; b) de rester à proximité de la complexité inhérente aux phénomènes relationnels ; c) de permettre un abord des aspects subjectifs et émotionnels qui sont très régulièrement mobilisés par la référence à un cas précis 5 .

Précisons :

a) que le travail effectué à partir des cas ne concerne jamais la sphère privée, mais cette " personnalité professionnelle du soignant » dont parle M. Balint ${ }^{6}$; b) qu'il nous est apparu, à ce stade des études, trop difficile et potentiellement générateur de résistances de travailler avec l'étudiant directement sur sa propre relation avec un malade. Le cas rapporté est donc utilisé comme induction au travail du groupe. Il ne s'agit pas, à la différence des groupes Balint ${ }^{7}$ d'interroger la relation de l'étudiant rapporteur du cas à son patient, mais la relation du groupe au cas, ceci pour en tirer des éléments d'enseignement.

\section{Le groupe}

Lintérêt du travail de groupe est connu : mise au contact d'autres manières de faire et de réagir, découverte d'une pluralité de réponses possibles plutôt que d'un modèle unique, efficacité pédagogique des contributions venant d'autres à la foi proches et différents, mobilisation des affects suscitant la réflexion ${ }^{8}$, etc. De plus, le groupe est pour ce domaine de formation un outil particulièrement intéressant en ce sens qu'il produit « un contenu relationnel ", permettant ainsi d'expérimenter " in vivo » certains des processus à l'œuvre dans la relation (prise de conscience des a priori, des identifications, etc.) ${ }^{9}$.

\section{Le binôme enseignant}

Les groupes sont animés par deux enseignants, l'un médecin généraliste (ou spécialiste), l'autre psychiatre, psychologue et/ou psychanalyste. Cette co-animation, médecin professionnel de la psychologie clinique a comme intérêt spécifique : a) d'instituer de fait cet « effet de double voix " (incarné par la double fonction), qui manifeste que la manière de percevoir une situation, tout comme les solutions qui sont proposées, ne sont pas univoques; b) de permettre de se démarquer de l'identification à «l'enseignant d'expérience », qu'il soit médecin ou professionnel de la psychologie clinique, supposé avoir les solutions ce qui, dans le domaine considéré, ne peut être qu'un leurre. Un dispositif de co-animation répond aussi à certaines contraintes inhérentes au contenu de la formation proposée. L'abord de questions où la subjectivité de chacun est en jeu (et comment en serait-il autrement pour la relation ?) contient en effet toujours le risque que l'animateur projette ses présupposés, voire sa propre problématique sur la situation. La co-animation favorise la prise de recul des formateurs, relativisant le point de vue de l'un par celui de l'autre, offrant ainsi une garantie qu'un enseignant ne fasse pas passer pour vérité ses a priori $^{10}$. Elle permet aussi - l'animation de tels groupes mobilisant toujours l'enseignant sur le plan émotionnel - que chaque formateur ne traduise pas sans discernement dans le groupe ses propres affects. Signalons que, pour tous les intervenants, une formation à l'animation de groupe et au travail sur la relation (participation à un groupe Balint ou à d'autres groupes de travail sur la relation médecinmalade, analyse personnelle, études de psychologie ou de psychiatrie, etc.) est requise. Tout nouvel enseignant inté- 
gré dans l'équipe est d'abord observateur pendant une année, puis associé à un enseignant d'expérience lors de ses premières années d'intervention.

\section{Lecture d'articles et écriture d'un mémoire de fin d'enseignement}

Le fait de lier explicitement la lecture d'articles à l'écriture d'un texte portant sur l'expérience d'enseignement et d'apprentissage constitue le quatrième outil pédagogique de notre programme de formation. L'introduction obligatoire de références aux articles du polycopié dans le mémoire de fin d'enseignement vise à la fois : a) l'acquisition et une intégration optimale des connaissances et des notions de base sur la relation médecin-malade (développées dans des articles), par un travail d'écriture orienté vers leur application à un cas clinique précis et à une expérience de groupe de formation ; b) le développement d'une réflexion personnelle et une relecture de ce qui s'est passé dans le groupe à la lumière des notions développées dans les articles du polycopié.

\section{Descriptif technique et justification des groupes de parole et de réflexion sur cas (premier module d'enseignement)}

Le fait que nous intervenions auprès de "jeunes étudiants " et que cet enseignement soit obligatoire pour tous détermine dans une large mesure le dispositif pédagogique proposé (accompagnement à la prise de parole, respect d'un cadre précis, attention au risque de déstabilisation des étudiants, etc.).

\section{Déroulement pratique}

\section{Première séance}

Le groupe se dispose assis sur les chaises en un cercle, sans interposition de tables. La consigne suivante est donnée aux étudiants : " chacun va en binôme avec son voisin, d'une part se présenter (nom, prénom), dire dans quel service hospitalier il est passé au semestre précédent et rapporter une situation qui l'a marqué ou qu'il pense intéressante de travailler dans le cadre d'un enseignement sur la relation médecin malade ». Chacun, alternativement, dispose de 10 minutes pour parler à l'autre. Ensuite, chaque étudiant présente son voisin au groupe et rapporte la situation qu'il a exposée. Un des enseignants anime le groupe pendant que l'autre prend des notes les plus précises sur les cas présentés. Dans l'après-cours, les deux enseignants se réunissent pour regrouper les situations évoquées en cinq thèmes (ce qui correspond aux cinq séances restantes) et choisissent celui qui sera travaillé la fois sui- vante. De manière idéale, chaque situation doit trouver sa place afin que tous les cas évoqués lors de cette première séance soient, d'une manière ou d'une autre, exploités avant la fin du module.

\section{Séances suivantes}

Il est proposé aux étudiants, à partir du thème retenu par les enseignants pour le cours, de revenir sur une des situations évoquées lors de la première séance en demandant à celui qui l'a vécue d'en restituer les grandes lignes. Le débat s'instaure ensuite entre les étudiants, sous la conduite des animateurs. Quand un cas a été suffisamment travaillé et/ou que l'intérêt s'en fait sentir, les enseignants proposent de passer à une autre situation : « le cas de X semblait poser des questions voisines (ou un peu différentes) ; pouvez-vous nous le rappeler ?".

Le rôle des enseignants consiste à : a) relancer les questions, souligner les éléments qui semblent rassembler le groupe et ceux sur lesquels portent des différences d'appréciation, faire réfléchir aux oppositions de réactions et de point de vue; b) aider le groupe à repérer quelques-uns uns des présupposés et des éléments non conscients sousjacents aux différentes positions exprimées ; c) souligner les évolutions personnelles ou du groupe au cours de la séance.

\section{Justification de l'intérêt des techniques employées et principes pédagogiques mis en ouvre}

La présentation deux à deux permet à tous les étudiants de «parler devant le groupe » dès cette première séance, favorisant notamment l'expression des plus timides (qui vont pouvoir d'abord s'exprimer face à un seul interlocuteur avant de s'exposer en groupe). L'expression est aussi facilitée par la position de " rapporteur du cas de l'autre ", plus aisée à occuper que celle qui consiste à parler en son nom propre et de son cas face au groupe.

Le tour de table complet dans la première séance permet : l'intégration de chacun dans le groupe; une prise de conscience que les difficultés et affects rencontrés par chacun lors de son passage à l'hôpital sont partagés par d'autres et que des situations voisines peuvent être perçues différemment; de produire un matériel très riche exploitable par simple rappel lors des séances suivantes.

L'apport des cas par les étudiants a l'intérêt : de rester au plus près des questions qu'ils se posent et de ce qu'ils ont vécu (ceci est essentiel pour susciter leur attention et leur réflexion et est particulièrement important pour la formation à la relation qui, quand elle s'éloigne de l'expérience concrète, fait régulièrement surgir chez les étudiants des résistances ${ }^{11}$ ); de permettre la verbalisation de ce qui est vécu par les étudiants dans une grande intensité émotion- 
nelle - parfois une souffrance - et de pacifier des situations perçues comme traumatiques ${ }^{12,13}$; de pouvoir « cheminer avec le groupe sans le devancer ». Il est ici important que les animateurs laissent au groupe le temps d'une élaboration et privilégient ses propres découvertes à celles qu'ils pourraient être tentés de fournir en tant qu'enseignants. Une certaine "réserve » est donc observée par les animateurs, une idée produite par le groupe étant mieux intégrée et plus efficace en terme de formation ${ }^{12}$. Ceci permet d'utiliser l'apport des autres participants (" des autres un peu comme nous, un peu différents " tels que le décrivait un étudiant dans son mémoire en 2003) pour l'apprentissage ; de laisser chacun avancer à son rythme, ce qui se réalise grâce à une démarche en trois temps [1) repérage des modes de réaction des autres et de leur diversité ; 2 ) interrogation sur ce qui motive ces réactions chez l'autre ; 3) questionnement sur ses propres modes de réaction en situation].

\section{Descriptif technique et justification du jeu de rôle (second module d'enseignement)}

\section{Déroulement pratique}

Première séance

Son déroulement comporte deux temps : 1) explication de la technique du jeu de rôle et présentation de ce qu'il peut apporter dans l'apprentissage de la relation. Le programme et les objectifs sont également largement explicités en précisant que l'on ne va juger ni les compétences médicales, ni la capacité théâtrale des participants ; 2) initiation au jeu de rôle par l'utilisation d'une courte vignette qui va permettre à tous les étudiants de participer à un premier jeu (exemple : «le patient qui vous fait un cadeau ", "le patient qui part sans payer »). Les étudiants vont successivement jouer cette situation deux à deux devant le groupe, l'un prenant la position du médecin, l'autre celle du malade. L'échange est limité à quelques répliques et l'on passe au doublet suivant.

\section{Séances suivantes}

Un schéma en trois temps est utilisé.

1. Préparation du jeu

Elle débute par la proposition par un enseignant d'une situation choisie dans la bibliothèque de jeux de rôles du DERPM. Le thème sur lequel porte le cas est présenté à l'ensemble du groupe, avec appel aux " acteurs » qui se désignent de façon libre. Les joueurs sortent de la salle avec un des enseignants qui prend à part le " patient " et lui propose le synopsis détaillé de son rôle. Il s'adresse de même ensuite au « médecin » mais en lui donnant simple- ment quelques indications indispensables, notamment les précisions techniques médicales sur lesquelles il pourra s’appuyer. Son rôle dans la relation n'est pas précisé et il doit improviser son attitude en fonction des problèmes rencontrés. Un exemple détaillé d'un jeu de rôle est fourni dans l'annexe 1. L'autre enseignant, resté dans la salle, sépare le groupe en deux parties, l'une " observant " le médecin, l'autre le malade; il engage avec le groupe une courte discussion sur la problématique posée par la situation travaillée.

2. Déroulement du jeu

Le jeu débute et va durer de 10 à 15 minutes. Les enseignants sont prêts à intervenir si l'un des joueurs est en difficulté ou encore pour relancer, d'une suggestion faite à l'oreille d'un des acteurs, un jeu qui s'enlise dans une impasse. Ils peuvent demander aux étudiants de rejouer ce qu'ils viennent d'interpréter en proposant éventuellement d'inverser les positions des joueurs.

3. Exploitation du jeu

Les animateurs interrogent d'abord les " acteurs " sur la manière dont ils ont vécu la situation et ce qu'ils en pensent. La parole est ensuite donnée aux étudiants ayant observé le jeu, en un tour de table dont les principes et la technique d'animation sont les mêmes que dans le premier module.

\section{Justification de l'intérêt des techniques employées et principes pédagogiques mis en œuvre}

Du fait des objectifs de la formation, le jeu de rôle utilisé est de type jeu de rôle d'exploration. Celui-ci est, comme nous l'avons décrit, employé dans sa forme classique, avec scène unique, simple ou suivie d'inversion et animation en trois étapes ${ }^{14}$. Dans ce type de jeu de rôle, « visant à explorer un aspect du savoir-être professionnel [...] le participant utilise la latitude disponible pour s'investir personnellement dans l'interprétation du jeu de rôle " ${ }^{14}$.

L'objectif spécifique de la première séance est de dédramatiser le fait de jouer devant les autres, ce qui est obtenu : en faisant jouer tous les participants ; en utilisant une vignette clinique n'entrainant pas trop d'implication ; en faisant l'économie d'une « exploitation » approfondie du jeu.

Pour les séances suivantes qui visent au repérage des éléments subjectifs et émotionnels présents dans la relation, le fait que nous nous adressions à de jeunes étudiants, que le jeu de rôle d'exploration sollicite l'implication personnelle du joueur, que la formation à la relation mêle irréductiblement formation professionnelle et personnelle ${ }^{5}$, rend nécessaire la référence à un cadre pédagogique précis, afin d'éviter des déstabilisations non contrôlées des étudiants ou les dérives vers l'intime.

Le cadre pédagogique du jeu de rôle dans notre dispositif 
de formation implique la constitution du groupe et la mise en place d'un climat de confiance préalable. Ceci est assuré grâce au travail réalisé dans le premier module. Les interventions des enseignants et des étudiants sont définies comme devant viser uniquement ce qui se passe dans la relation professionnelle et non dans la sphère privée. Il n'y a donc pas « d'interprétation personnelle » de la part des animateurs qui, de plus, le précisent aux étudiants et veillent à ce que ce cadre soit maintenu pendant les discussions. Les consignes insistent sur le fait que nous nous situons dans le " comme si ». Il ne s'agit donc pas, comme dans un psychodrame ${ }^{15}$, de reconstituer une scène personnellement vécue par un étudiant ; il n'y a pas de "restitution " ou de " reconstruction ». Il s'agit, à partir d'une situation exemplaire construite à l'avance, de faire travailler le groupe sur les problématiques relationnelles que le jeu va mettre en évidence. Le scénario du jeu de rôle est choisi et préparé avant la séance par les enseignants. Cette " préparation » du jeu permet aux enseignants : de réfléchir à la succession des situations à proposer; d'utiliser au mieux la valeur inductrice des cas proposés, cela en fonction de l'évolution du groupe, de son questionnement et des objectifs pédagogiques suivis. Le fait que le scénario soit écrit offre une " protection » quant à l'implication personnelle mobilisée par le fait de jouer et constitue une garantie face au risque de déstabilisation de l'étudiant.

L'écriture du cas sera toujours assez précise pour le "rôle patient » qui, non cadré, expose davantage à des débordements vers le domaine du privé. Le respect du droit à la non-participation au jeu est garanti. Il n'y a aucune obligation de jouer. Ainsi chaque refus individuel, soit à l'égard de la méthode, soit à l'égard de telle ou telle situation, doit être respecté.

\section{Le mémoire de fin de formation}

En fin de formation, les étudiants rédigent un mémoire. Ils sont informés que celui-ci ne sera pas apprécié à l'aide d'un score mais que ce texte devra répondre à quatre critères pour être validé :1) rapporter un ou des cas cliniques abordés (évaluation de l'attention de l'étudiant et de sa capacité d'écoute dans le groupe ) ; 2) analyser le cas et décrire les réactions du groupe lors du travail en commun (évaluation de la capacité d'analyse et de compréhension d'une situation clinique) ; 3) décrire l'évolution de ses propres conceptions à l'occasion du cas choisi et/ou sur l'ensemble des deux modules (évaluation de la capacité de questionnement personnel et d'auto-examen $\left.{ }^{16}\right)$; 4) faire un lien avec les articles ou la bibliographie proposée (contrôle de l'effectivité de la lecture et de la capacité à utiliser des références dans une argumentation).

Ce temps consacré à l'élaboration personnelle d'un écrit vise : à favoriser la réflexion de chaque étudiant sur ce qui s'est passé dans le "stage en groupe »; à mettre ceci en lien et en débat critique avec des travaux en cette matière, dont le recueil d'articles qui leur est remis donne un aperçu; à permettre l'acquisition et l'appropriation des connaissances et des notions de base contenues dans ces articles par leur application à un contexte de formation et à un cas clinique précis. La bibliographie remise aux étudiants sous forme d'un polycopié est volontairement limitée en volume de manière à permettre aux étudiants de lire tous les textes qu'elle contient. Cinq textes sont proposés : un extrait du livre de Georges Canguilhem, Etudes d'Histoire et de Philosophie des Sciences ${ }^{2}$; l'article de Lucien Israël de l'Encyclopaedia Universalis: "la relation malade médecin "; un chapitre du livre de Michael Balint, Techniques psychothérapeutiques en médecine : "le métier de comprendre autrui ${ }^{17}$; Un article d'Eric Galam : "la médecine s'exerce dans un contexte de représentations et de croyances", extrait du livre de la conférence permanente de médecine générale, Pédagogie de la relation thérapeutique en médecine générale ${ }^{18}$; un article de Ginette Rimbault : "Ya-t-il une théorie de la relation " paru dans la revue Champ Psychosomatique ${ }^{19}$.

\section{Evaluation préliminaire du dispositif d'enseignement}

Plusieurs sources d'information permettent de porter une appréciation préliminaire concernant la perception par les étudiants et l'impact du programme d'enseignement d'initiation à la relation médecin-malade, tel que nous l'avons expérimenté dans notre institution : 1) un recueil du degré de satisfaction portant sur les contenus, les méthodes pédagogiques et la qualité de l'intervention des enseignants, effectué à l'aide d'un questionnaire ; 2) le recueil de l'avis des étudiants sur l'apport de l'enseignement à partir des réponses à un questionnaire portant sur des éléments caractéristiques de la relation médecin malade ou des situations typiques de la pratique médicale. Même s'il convient de souligner les limites d'une telle évaluation réalisée, puisque les résultats quantifiés ne concernant que l'appréciation du degré de satisfaction des étudiants et leur perception quant à l'apport de la formation, on relève un taux de satisfaction global très positif : dans tous les groupes et quels que soient les items (adaptation du contenu à la pratique, méthodes pédagogiques, intervention des enseignants), le pourcentage d'étudiants satisfaits est supérieur à $80 \%$. L'appréciation positive globale 
de l'enseignement varie selon les groupes de 85 à $100 \%$. Leur estimation de l'apport de l'enseignement, tous items confondus, est quantifiée par les étudiants à 6,8 sur 10 , ce qui peut être considéré comme satisfaisant. L'intérêt de ces réponses est limité par le fait qu'il ne s'agit que de la mesure de leur perception quant à l'évolution de leurs compétences (mieux comprendre : les malades, la nature de vos réactions face aux patients, ce que l'attitude du médecin peut induire chez le patient,...) et non d'une mesure objective de cette évolution qui nécessiterait l'utilisation de méthodes d'évaluation différentes et plus complexes à mettre en œuvre. Si ces deux questionnaires ne permettent évidemment pas de mesurer l'efficacité de l'enseignement quant à l'acquisition de connaissances, de compétences ou d'habiletés, elles mettent en évidence l'intérêt que les étudiants trouvent à cet enseignement, son adaptation à leurs besoins ressentis et sa faisabilité. La bonne homogénéité des réponses entre les groupes est à souligner. Concernant les mémoires des étudiants aucune grille d'évaluation systématique permettant de produire des résultats sous forme de score n'est actuellement en place. Signalons que pour l'équipe pédagogique, l'analyse et la compréhension des situations cliniques rapportées sont satisfaisantes et que la qualité de l'utilisation des références à la bibliographie est tout à fait remarquable. Les quatre critères nécessaires à la validation sont présents dans l'immense majorité des mémoires.

\section{Conclusion}

Responsables d'enseignements, pouvoirs publics, associations de malades s'accordent sur l'importance d'une formation à la relation des étudiants en médecine. Le programme de formation « initiation à la relation médecin malade », mis en place à la faculté de médecine de Créteil (Université de Paris XII) est présenté dans cet article en insistant sur les méthodes utilisées et leurs justifications en termes pédagogiques. Le travail sur cas clinique issus de l'expérience des étudiants, en situation groupale, employant les méthodes du groupe de parole et du jeu de rôle, co-animé par un binôme enseignant constitué par un médecin et un professionnel de psychologie clinique, donnant lieu à la rédaction d'un mémoire avec référence obligatoire à des articles sélectionnés, en constituent les principaux éléments. Les démarches d'évaluation mettent en évidence l'excellente appréciation des étudiants et la faisabilité de l'enseignement, mais ne permettent pas d'apprécier réellement les apprentissages développés. Cette expérience donne matière à un certain nombre de propositions générales sur l'enseignement de la relation médecin-malade dont la fonction est de participer à la recherche actuellement en cours sur ce plan.

\section{Annexe 1 \\ Exemple de jeu de rôle utilisé La relation mère-enfant}

\section{La situation}

Une dame, 34 ans, vient pour la première fois consulter le médecin pour Paul, son petit garçon de 5 ans. Il dort mal, vomit souvent ses repas et se plaint d'avoir mal au ventre. Elle vient d'emménager en proche banlieue parisienne, après une séparation houleuse de son mari, avec qui elle vivait depuis l'âge de 21 ans et qui avait entrepris de longues études. Pendant ce temps, son propre salaire assurait le quotidien. Depuis, son mari a une belle situation. Il y a cinq ans ils se sont mariés quand elle attendait un enfant et elle a cessé de travailler.

Ils habitaient à Paris dans un grand appartement très confortable. Peu après la naissance de Paul, les relations du couple se sont détériorées. C'est lorsqu'elle a appris qu'il la trompait avec une jeune femme de 25 ans qu'elle a décidé de le quitter.

En attendant que le divorce soit prononcé, elle et son fils vivent dans un appartement de trois pièces. Elle a retrouvé un emploi et inscrit Paul dans une nouvelle école. Il reste à la cantine et à la garderie jusqu'à son retour du travail. Depuis, le comportement de Paul a changé, il n'est plus vif et enjoué comme avant. Le soir, il refuse d'aller au lit. La nuit, il se réveille et vient dans le lit de sa mère.

\section{La mère}

Pendant la consultation, elle a tendance à surprotéger Paul et à accaparer la parole. De temps en temps elle se penche tendrement vers lui. Elle est un peu agitée et inquiète. Elle ne parle que des troubles digestifs de Paul, sans évoquer leur nouvelle situation avant que le médecin ne l'y incite.

\section{L'enfant}

Il a 5 ans et a "mal au ventre ". Il est venu chez le médecin avec son ours. Il suce son pouce, renfrogné, il baisse la tête et s'accroche à sa mère. Il a déménagé et changé d'école, son institutrice ne lui plaît pas. Il voit son père tous les 15 jours.

\section{Le médecin}

Il voit cette dame et son enfant pour la première fois. Il a minutieusement examiné l'enfant et n'a rien trouvé d'inquiétant. 


\section{Références}

1. Richard C, Lussier M-T (Dir.). La communication professionnelle en santé. Saint Laurent (QC) : ERPI, 2005.

2. Canguilhem G. Etudes d'Histoire et de Philosophie des Sciences. Paris : Vrin, 1983.

3. Barrier J-H, Brazeau-Lamontagne L, Pottier P, Boutoille D. Comparaison des compétences médicales éthiques des étudiants de $3^{e}$ et de $\sigma^{e}$ année en stage hospitalier en médecine interne. Rev Med Interne 2005;26:128-36.

4. Balint M. Techniques psychothérapeutiques en médecine. Paris : Payot, 1966.

5. Sapir M. Formation à la relation soignant-soigné : mythe et réalité. Grenoble : La Pensée Sauvage, 1989.

6. Balint M. Le médecin, son malade et la maladie. Paris : Payot, 1960.

7. Even G. Comment fonctionne un groupe Balint ? Revue du Praticien - Médecine générale 2001;15: 564-5.

8. Maccio C. Techniques de la vie en groupe. Lyon : Chronique sociale, 1997.

9. Anzieu D, Martin J-Y. La dynamique des groupes restreints. Paris : PUF, 1968.

10. Anzieu D. Le groupe et l'inconscient, l'imaginaire groupal. Paris : Dunod, 1984.
11. Even $G$. Former les étudiants en médecine à la relation. Champ Psychosomatique 2001;22:133-42.

12. Ruzniewski M. Face à la maladie grave. Paris : Dunod, 1995.

13. Kent GG. Medical students' reaction to a nursing attachment scheme. Med Educ 1991;25:23-31.

14. Girard G, Clavet D, Boulé R. Planifier et animer un jeu de rôle profitable pour l'apprentissage. Pédagogie Médicale 2005;3:178-85.

15. Leutz G-A. Mettre sa vie en scène, le psychodrame. Paris : Epi, 1985.

16. Morin E. La méthode. Ethique (tome 6). Paris : Seuil, 2004.

17. Balint M. Techniques psychothérapeutiques en médecine. Paris : Payot, 1966.

18. Conférence Permanente de la Médecine Générale. Pédagogie de la relation thérapeutique en médecine générale (Apports théoriques), Paris : Editoo.com, 2003.

19. Rimbault G. Y a-t-il une théorie de la relation ? Champ psychosomatique, Disponible auprès de : La Pensée Sauvage 12, place Notre-Dame 38000 Grenoble France.

Manuscrit reçu le 4 mars 2004 ; commentaires éditoriaux formulés aux auteurs le 7 octobre 2004 et le 5 juillet 2006 ; accepté pour publication le 7 juillet 2006. 